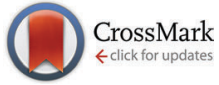

Cite this: Chem. Commun., 2015, 51, 9995

Received 7th March 2015, Accepted 11th May 2015

DOI: $10.1039 / \mathrm{c} 5 \mathrm{cc} 01937 \mathrm{k}$

www.rsc.org/chemcomm

\title{
Visible light-induced singlet oxygen-mediated intracellular disassembly of polymeric micelles co-loaded with a photosensitizer and an anticancer drug for enhanced photodynamic therapy $\dagger$
}

\author{
Gurusamy Saravanakumar, Junseok Lee, Jihoon Kim and Won Jong Kim*
}

\begin{abstract}
Herein, we report a biocompatible amphiphilic block copolymer micelle bearing a singlet oxygen-sensitive vinyldithioether cleavable linker at the core-shell junction, which undergoes singlet oxygenmediated photocleavage in the presence of visible light. The micelle facilitates the light-responsive release of singlet oxygen and an anticancer drug for enhanced photodynamic therapy.
\end{abstract}

Photodynamic therapy (PDT) is emerging as a promising treatment modality for several cancers and other non-malignant diseases owing to its unique advantages such as non-invasiveness and less systemic toxicity. ${ }^{1}$ This modality works by administering a photosensitizer (PS) and then activating it with a light of specific wavelength to generate reactive oxygen species, primarily singlet oxygen $\left({ }^{1} \mathrm{O}_{2}\right)$, which induce apoptosis or necrosis of the tumor cells. Despite its significant advantages, however, the clinical potential of PDT is limited by factors such as poor aqueous solubility of PSs and nonspecific accumulation of PSs at the normal tissues. To surmount these issues, several nanocarrier platforms such as liposomes, ${ }^{2}$ polymeric micelles, ${ }^{3}$ dendrimers, ${ }^{4}$ and self-assembled polymeric nanoparticles ${ }^{5}$ have been investigated. Another important concern of PDT is the depletion of ambient tumor oxygen through rapid and continuous photochemical conversion of ${ }^{3} \mathrm{O}_{2}$ to ${ }^{1} \mathrm{O}_{2}$ at high fluence rates, which can cause acute local hypoxic conditions and disrupt the photodynamic process, thereby reducing the treatment effectiveness. ${ }^{6}$

Integration of PDT with other treatment modalities such as photothermal therapy ${ }^{7}$ or chemotherapy ${ }^{8}$ holds great promise to circumvent the above critical limitation, because such a combination therapy produces additive or synergistic therapeutic efficacy by targeting different cellular or molecular pathways. It is particularly gratifying to note that the results from recent preclinical studies have also indicated that combining PDT with

Center for Self-assembly and Complexity, Institute of Basic Science (IBS), and Department of Chemistry, Pohang University of Science and Technology,

Pohang 790-784, Republic of Korea. E-mail: wjkim@postech.ac.kr;

Fax: +82-279-3399; Tel: +82-57-279-2104

$\dagger$ Electronic supplementary information (ESI): Experimental details, supplementary figures and tables. See DOI: 10.1039/c5cc01937k chemotherapy can improve anticancer activity by overcoming anticancer drug resistance ${ }^{9}$ which is a major impediment to the success of chemotherapy. Considering highly liphophilic and molecularly diverse characteristics of both PSs and anticancer drugs, however, the development of smart and efficient carriers for the co-delivery of PSs and anticancer drugs is crucial to realize the full potential of the combination therapy.

For such co-delivery, we envisioned that polymeric micelles integrated with photo-responsive moieties could be a potential carrier. Firstly, its core-shell structures could readily encapsulate multiple poorly water-soluble active agents into the hydrophobic core with high loading capacity. Secondly, without any additional trigger mechanism, the co-loaded anticancer drugs can be effectively released in the tumor cell to exert their therapeutic effects because light is an integral part of the PDT process. Although light-responsive micellar systems have been developed by incorporating photochromic moieties such as azobenzene, ${ }^{10}$ 2-diazo-1,2-napthoquinone, ${ }^{11} \mathrm{O}$-nitrobenzyl ${ }^{12}$ or coumarin ${ }^{13}$ into their structure, unfortunately, most of them respond only to the harmful UV light or multi-photon excitation that requires enormously high laser power. Moreover, these systems are mostly based on non-degradable polymeric compositions with limited biocompatibility. Thus, there is an increasing need to develop safe, low toxic and more efficient carrier systems that could respond to the biomedically benign visible or near-infra-red light. It should also be emphasized that, to date, no significant effort has been made to the development of rationally designed light-responsive carriers for dual-drug delivery.

In this study, we have designed and explored a novel biocompatible visible-light responsive amphiphilic poly(ethylene glycol)-block-poly(caprolactone) copolymer $\left({ }^{1} \mathrm{O}_{2}\right.$-PEG- $b$-PCL) capable of forming micelles by incorporating an ${ }^{1} \mathrm{O}_{2}$-sensitive vinyldithioether linker between the hydrophilic PEG and the hydrophobic and biodegradable PCL block. The double bond of the vinyldithioether linker can readily react with ${ }^{1} \mathrm{O}_{2}$ to form a dioxetane intermediate, which is unstable and spontaneously cleaved into fragments. Therefore, unlike other conventional photo-responsive micelles that demand direct absorption of 

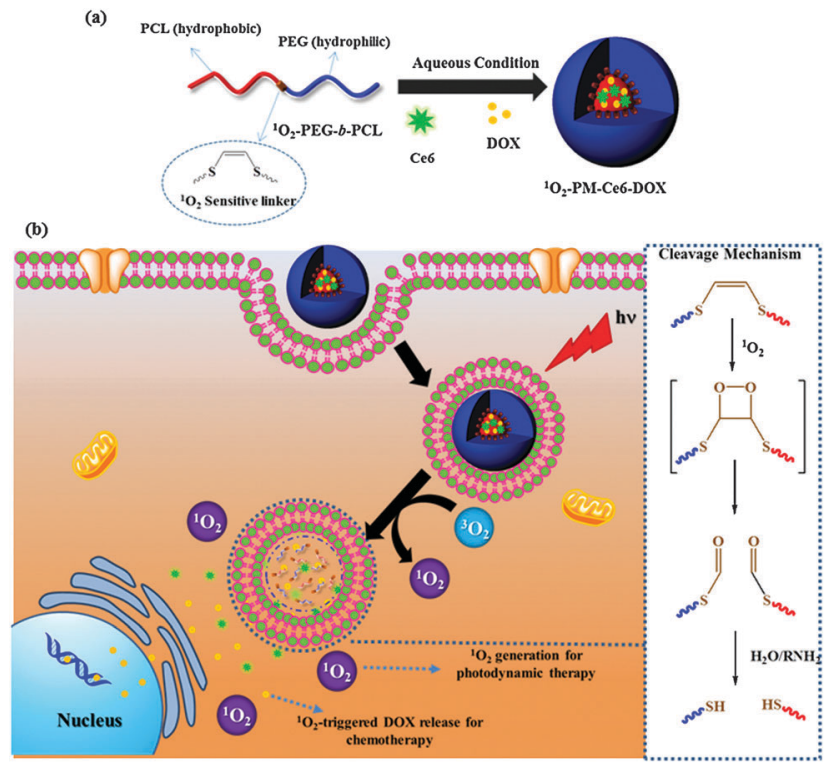

(c)

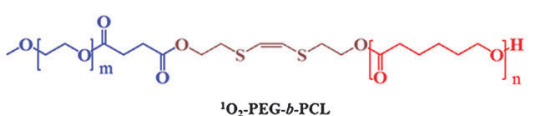

Scheme 1 (a) Schematic illustration of (a) the formation of Ce6 and DOX co-loaded micelles $\left({ }^{1} \mathrm{O}_{2}\right.$-PM-Ce6-DOX), (b) cellular uptake and visible lighttriggered ${ }^{1} \mathrm{O}_{2}$-mediated intracellular co-delivery of $\mathrm{Ce} 6$ and DOX for combination chemo and photodynamic therapy; and (c) chemical structure of the ${ }^{1} \mathrm{O}_{2}$-PEG-b-PCL copolymer.

UV light, our micellar system exploits the ${ }^{1} \mathrm{O}_{2}$ generated by visible light during PDT as a trigger to disassemble the nanostructure and release a co-loaded drug in the tumor cell for effective therapy (Scheme 1). Furthermore, the cleaved fragments do not produce any toxic species like other photodegradable moieties. The potential of the micelle as a dual-drug carrier for enhanced photodynamic therapy was investigated by physical co-encapsulation of a PS, chlorine e6 (Ce6), and a hydrophobic anticancer drug, doxorubicin (DOX).

The synthetic strategy of a biocompatible ${ }^{1} \mathrm{O}_{2}$-PEG- $b$-PCL copolymer involves three steps (as illustrated in Fig. S1, ESI $\dagger$ ). First, a ${ }^{1} \mathrm{O}_{2}$-sensitive vinyldithioether linker was synthesized following a reported method, ${ }^{14}$ and its ${ }^{1} \mathrm{H}$-NMR spectrum (Fig. S2, ESI $\dagger$ ) was in good agreement with the literature. Second, a PEG macroinitiator $\left({ }^{1} \mathrm{O}_{2}\right.$-PEG-OH) bearing an ${ }^{1} \mathrm{O}_{2}$-sensitive linker was synthesized by coupling a carboxylated PEG to the excess of as-synthesized linker via Steglich esterification. The successful conjugation was confirmed by the characteristic cis-alkene peak $(\sim \mathrm{SCH}=\mathrm{CHS} \sim)$ at $\delta 6.12 \mathrm{ppm}$ from the linker and the PEG peaks $\left(\mathrm{CH}_{3}-\mathrm{O}-\right.$ at $\delta 3.36 \mathrm{ppm}$, and $-\mathrm{CH}_{2} \mathrm{CH}_{2}-$ at $\left.\delta 3.62 \mathrm{ppm}\right)$ in the ${ }^{1} \mathrm{H}-\mathrm{NMR}$ spectrum of ${ }^{1} \mathrm{O}_{2}$-PEG-OH (Fig. S3a, ESI $\dagger$ ). Third, the ${ }^{1} \mathrm{O}_{2}$-PEG- $b$-PCL copolymer was synthesized by ring-opening polymerization of the $\varepsilon$-caprolactone $(\varepsilon-\mathrm{CL})$ monomer using the ${ }^{1} \mathrm{O}_{2}$-PEG-OH as a macroinitiator in the presence of $\mathrm{Sn}(\mathrm{Oct})_{2}$ as a catalyst. The chemical structure of the block copolymer was confirmed by ${ }^{1} \mathrm{H}$-NMR spectroscopy (Fig. S3b, ESI $\dagger$ ), in which all the resonance peaks corresponding to the PEG and PCL segments were observed. Further, the composition of the copolymer was estimated by comparing the integration ratios of the peaks at $3.35 \mathrm{ppm}$ from the methyl protons $\left(\mathrm{CH}_{3}{ }^{-}\right)$of PEG and the peak at $4.03 \mathrm{ppm}$ from the methylene protons $\left(-\mathrm{CH}_{2}-\mathrm{O}-\right)$ of PCL segments. GPC analysis of the block copolymer showed a unimodal molecular weight distribution (Fig. S4, ESI $\dagger$ ). For comparison, a control C-PEG- $b$-PCL copolymer of similar composition without the linker was also synthesized. The compositions of these block copolymers are summarized in Table S1 (ESI $\dagger$ ).

Owing to its amphiphilicity, the ${ }^{1} \mathrm{O}_{2}$-PEG- $b$-PCL copolymer can form a self-assembled micellar structure $\left({ }^{1} \mathrm{O}_{2}\right.$-PM) under aqueous conditions. The dynamic light scattering measurements of micelles showed unimodal size distribution with an average particle size of $40 \pm 3.1 \mathrm{~nm}$ and the TEM image indicated that they have a spherical morphology (Fig. S5, ESI $\dagger$ ). Since the ${ }^{1} \mathrm{O}_{2}$-PM is incorporated with a ${ }^{1} \mathrm{O}_{2}$-sensitive linker, first we investigated whether the ${ }^{1} \mathrm{O}_{2}$-PEG- $b$-PCL copolymer can undergo visible-light induced ${ }^{1} \mathrm{O}_{2}$-mediated photocleavage. To investigate the photocleavage, the ${ }^{1} \mathrm{H}-\mathrm{HMR}$ spectra of the block copolymer solution containing $5 \mathrm{wt} \% \mathrm{Ce} 6$ without and with exposure to laser irradiation $\left(30 \mathrm{~mW} \mathrm{~cm}{ }^{-2}, 3 \mathrm{~h}\right)$ were recorded. As shown in Fig. S6a (ESI $\dagger$ ), the characteristic cis-alkene peak at $\delta 6.1 \mathrm{ppm}$ of the linker completely disappeared, whereas all the characteristic peaks of the polymers were observed without laser irradiation. This result was further supported by the GPC analysis (Fig. S6b, ESI $\dagger$ ), which showed the appearance of low molecular weight traces corresponding to the ${ }^{1} \mathrm{O}_{2}$-PEGmacroinitiator for the laser irradiated polymer. The above results clearly indicate that the block copolymer fragments into their two constituent blocks under laser irradiation.

After confirming the ${ }^{1} \mathrm{O}_{2}$-mediated photocleavage of the ${ }^{1} \mathrm{O}_{2}$-PEG- $b$-PCL copolymer, its potential as a carrier for co-delivery of a PS and an anticancer drug was investigated by encapsulating Ce6 and DOX via the dialysis method. For comparison, micelles loaded with only Ce6 were also prepared using the same method. The drug loading contents and loading efficiencies of these micelles are summarized in Table S2 (ESI $\dagger$ ). The size of Ce6 and DOX co-loaded micelles ( $\left({ }^{1} \mathrm{O}_{2}\right.$-PM-Ce6-DOX, Fig. 1a) was higher than Ce6-loaded micelles $\left({ }^{1} \mathrm{O}_{2}\right.$-PM-Ce6, Fig. S7a, ESI $\left.\dagger\right)$, indicating that the co-loading of Ce6 and DOX in the hydrophobic core increases the volume of micelles. The TEM images showed spherical morphologies for both ${ }^{1} \mathrm{O}_{2}$-PM-Ce6-DOX (Fig. 1b) and ${ }^{1} \mathrm{O}_{2}$-PM-Ce6 (Fig. S7b, ESI $\dagger$ ), although the size obtained by TEM is smaller than the DLS measurement due to the non-hydrated state. The size distributions of the micelles prepared using the block polymer without the ${ }^{1} \mathrm{O}_{2}$-linker are shown in Fig. S7c (ESI $\dagger$ ). To investigate whether ${ }^{1} \mathrm{O}_{2}$-PM-Ce6-DOX micelles can be disassembled by visible light, the TEM image of the micelles was recorded after exposing them to laser light irradiation ( $660 \mathrm{~nm}, 50 \mathrm{~mW} \mathrm{~cm}^{-2}, 3 \mathrm{~h}$ ). As expected, compared to the spherical micelles before irradiation, highly degraded irregular aggregated micelles were observed after laser irradiation (Fig. 1c). This clearly indicates that light-induced ${ }^{1} \mathrm{O}_{2}$-mediated photocleavage of the linker could disrupt the micellar structures by disturbing the hydrophilic-liphophilic balance. The UV-vis spectrum of ${ }^{1} \mathrm{O}_{2}$-PM-Ce6 showed a similar characteristic Soret band and Q-band absorption of free Ce6, indicating that the Ce6 in 

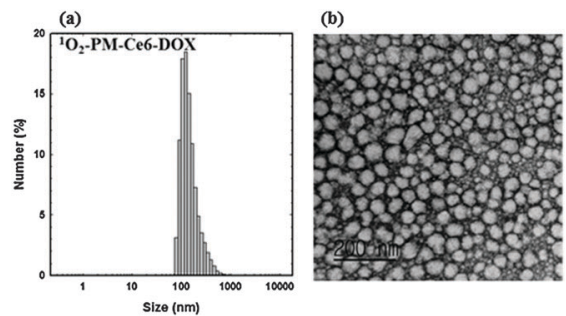

(c)

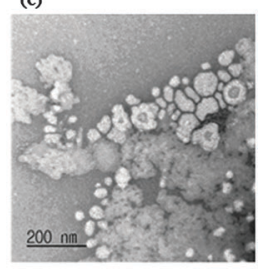

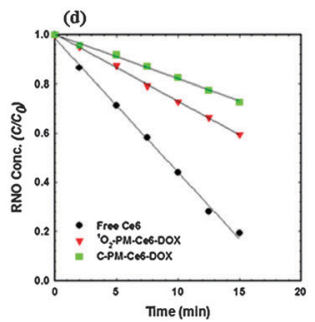

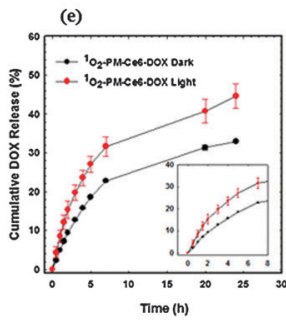

Fig. 1 (a) DLS size distribution, and TEM images of ${ }^{1} \mathrm{O}_{2}-\mathrm{PM}-\mathrm{Ce} 6-\mathrm{DOX}$ micelles (b) before and (c) after laser irradiation (scale bar is $200 \mathrm{~nm}$ ). (d) ${ }^{1} \mathrm{O}_{2}$ generation of ${ }^{1} \mathrm{O}_{2}$-PM-Ce6-DOX micelles determined by RNO as a sensor. (e) Visible light-triggered in vitro DOX release.

micelles existed in non-aggregated form (Fig. S8, ESI $\dagger$ ). The monomeric form is very important to ensure the photo-physical characteristic of Ce6 for efficient ${ }^{1} \mathrm{O}_{2}$ generation. The ${ }^{1} \mathrm{O}_{2}-$ generation efficiency of ${ }^{1} \mathrm{O}_{2}$-PM-Ce6-DOX was evaluated using the $\mathrm{N}, \mathrm{N}$-dimethyl-4-nitrosoaniline (RNO)-histidine colorimetric assay. ${ }^{15}$ In this assay, ${ }^{1} \mathrm{O}_{2}$ generated from the photoexcited sensitizer reacts with the imidazole moiety and forms a transient complex that bleaches the RNO molecules. Thus, the amount of ${ }^{1} \mathrm{O}_{2}$ can be directly correlated with a decrease in RNO absorbance in the UV-vis spectrum. Fig. 1d shows the RNO concentration as a function of time for free Ce6, ${ }^{1} \mathrm{O}_{2}$-PM-Ce6-DOX and C-PM-Ce6DOX. Upon irradiation, free $\mathrm{Ce} 6$ showed a sharp decrease in RNO concentration, indicating rapid ${ }^{1} \mathrm{O}_{2}$ generation. Although micelles showed a slow decrease in absorbance compared to the free Ce6, ${ }^{1} \mathrm{O}_{2}$-PM-Ce6-DOX showed a slightly fast decrease in RNO concentration as compared to C-PM-Ce6-DOX. This increase in ${ }^{1} \mathrm{O}_{2}$ generation efficiency of ${ }^{1} \mathrm{O}_{2}$-PM-Ce6-DOX could be due to the disassembly of micelles under laser irradiation, which releases the Ce6 molecules in monomeric form. Next, the drug release behaviour of the ${ }^{1} \mathrm{O}_{2}$-PM-Ce6-DOX was investigated in PBS (pH 7.4) with and without light. The cumulative release percentage of DOX loaded in micelles versus time is shown in Fig. 1e. The DOX release rates were accelerated in the presence of light irradiation compared to the dark conditions. The fast DOX release from the micelles was most likely due to the degradation micelles by the photocleavage of the vinyldithioether bonds. These results suggest that micelles incorporated with a ${ }^{1} \mathrm{O}_{2}$-sensitive linker can effectively release co-loaded drugs under photodynamic conditions at the target cells, which may improve the overall therapeutic efficacy.

As designed, the light-responsive release of DOX from ${ }^{1} \mathrm{O}_{2}$-PMCe6-DOX was deemed to exhibit the spatio-temporal controlled synergistic anticancer effects. Therefore, the therapeutic potential of ${ }^{1} \mathrm{O}_{2}$-PM-Ce6-DOX was evaluated in vitro. Irrespective of light on/off, the ${ }^{1} \mathrm{O}_{2}$-PM and C-PM micelles demonstrated insignificant
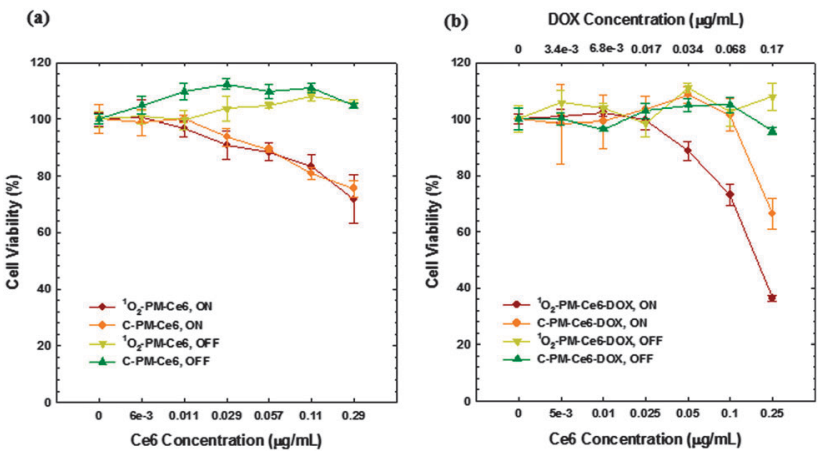

Fig. 2 Laser-induced cellular photo-toxicity of (a) ${ }^{1} \mathrm{O}_{2}-\mathrm{PM}-\mathrm{Ce} 6$ and C-PM$\mathrm{Ce} 6$ micelles, and (b) ${ }^{1} \mathrm{O}_{2}-\mathrm{PM}-\mathrm{Ce} 6-\mathrm{DOX}$ and $\mathrm{C}$-PM-Ce6-DOX micelles in PC3 cell lines.

cytotoxicity due to the biocompatibility of PEG and PCL (Fig. S9, ESI $\dagger$ ). In addition, no significant toxicity was observed with ${ }^{1} \mathrm{O}_{2}-$ PM-Ce6 and C-PM-Ce6 micelles under dark conditions (Fig. 2a). However, ${ }^{1} \mathrm{O}_{2}$-PM-Ce6 and C-PM-Ce6 micelles showed slight cytotoxicity under light irradiation, which was ascribed to the cytotoxic effects of ${ }^{1} \mathrm{O}_{2}$ generated from light-activated Ce6 (Fig. 2a). In the cases of Ce6 and DOX loaded micelles under dark conditions, cell viability was retained above $95 \%$ for micellar concentrations of up to $5 \mu \mathrm{g} \mathrm{mL} \mathrm{m}^{-1}$. The insignificant toxicity of DOX-releasing micelles was due to the lower concentration of DOX compared with general DOX doses ${ }^{16}$ for the in vitro test. Although each of ${ }^{1} \mathrm{O}_{2}$ and DOX released from the micelles could not exert the effective cytotoxic effects on the cancer cells, the combinatorial use of them showed the significant anticancer efficiency. Especially, under light irradiation, the ${ }^{1} \mathrm{O}_{2}$-PM-Ce6-DOX micelle showed the most efficient therapeutic effects compared to the C-PM-Ce6-DOX micelle (Fig. 2b). To demonstrate the potential of the micelles for intracellular delivery, cellular uptake and intracellular release behaviour of DOX from the micelles were evaluated using confocal laser scan microscopy. As shown in Fig. 3, the strongest fluorescence in the nucleus was observed in the cells incubated with ${ }^{1} \mathrm{O}_{2}$-PM-Ce6-DOX micelles exposed to light compared to the dark conditions and control micelles, which could be attributed to the enhanced release of DOX by ${ }^{1} \mathrm{O}_{2}$-responsive destruction of the micelle. Therefore, it can be concluded that the ${ }^{1} \mathrm{O}_{2}$-PM-Ce6-DOX micelle showed the potential not only for reducing the unintended cytotoxic effects

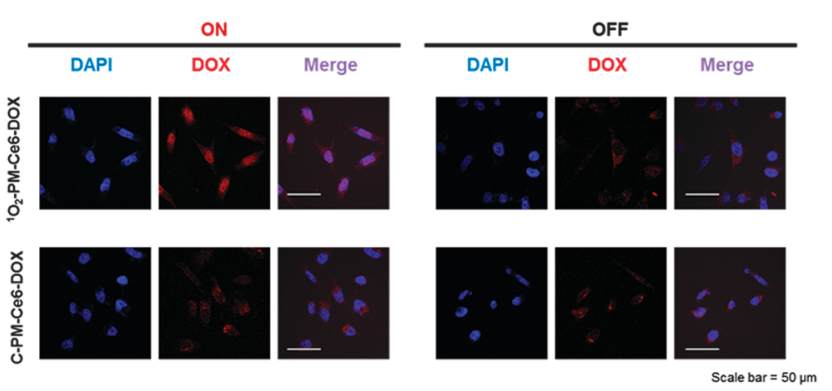

Fig. 3 Intracellular visible light-triggered release of DOX from ${ }^{1} \mathrm{O}_{2}-\mathrm{PM}$ Ce6-DOX micelles observed by CLSM using HeLa Cells. The micelles without the vinyldithioether linker (C-PM-Ce6-DOX) were used as control. 
on the normal tissues during blood circulation, but also for exhibiting the effective anticancer effects only on tumours.

In summary, visible light-triggered intracellular co-delivery of an anticancer drug and a PS using biocompatible polymeric micelles bearing vinyldithioether as a ${ }^{1} \mathrm{O}_{2}$ cleavable linker is realized for more effective photodynamic therapy. Given the high biocompatibility and ability to release drugs under photodynamic therapy conditions, the micellar system developed in this study could be a promising carrier for co-delivery of hydrophobic therapeutic drugs and PSs.

This work was supported by the Research Center Program of IBS (Institute for Basic Science) in Korea (CA1203-02).

\section{Notes and references}

1 (a) D. E. J. G. J. Dolmans, D. Fukumura and R. K. Jain, Nat. Rev. Cancer, 2003, 3, 380; (b) T. J. Dougherty, C. J. Gomer, B. W. Henderson, G. Jori, D. Kessel, M. Korbelik, J. Moan and Q. Peng, J. Natl. Cancer Inst., 1998, 90, 889; (c) I. J. Macdonald and T. J. Dougherty, J. Porphyrins Phthalocyanines, 2001, 5, 105.

2 A. S. L. Derycke and P. A. M. de Witte, Adv. Drug Delivery Rev., 2004, 56, 17.

3 (a) C. F. van Nostrum, Adv. Drug Delivery Rev., 2004, 56, 9; (b) B. Li, E. H. Moriyama, F. Li, M. T. Jarvi, C. Allen and B. C. Wilson, Photochem. Photobiol., 2007, 83, 1505; (c) H. Ding, B. D. Sumer,
C. W. Kessinger, Y. Dong, G. Huang, D. A. Boothman and J. Gao, J. Controlled Release, 2011, 151, 271.

4 N. Nishiyama, Y. Morimoto, W.-D. Jang and K. Kataoka, Adv. Drug Delivery Rev., 2009, 61, 327.

5 S. J. Lee, K. Park, Y.-K. Oh, S.-H. Kwon, S. Her, I.-S. Kim, K. Choi, S. J. Lee, H. Kim, S. G. Lee, K. Kim and I. C. Kwon, Biomaterials, 2009, 30, 2929.

6 B. W. Henderson, T. M. Busch, L. A. Vaughan, N. P. Frawley, D. Babich, T. A. Sosa, J. D. Zollo, A. S. Dee, M. T. Cooper, D. A. Bellnier, W. R. Greco and A. R. Oseroff, Cancer Res., 2000, 60, 525.

7 (a) B. Jang, J.-Y. Park, C.-H. Tung, I.-H. Kim and Y. Choi, ACS Nano, 2011, 5, 1086; (b) M. Zhang, T. Murakami, K. Ajima, K. Tsuchida, A. S. D. Sandanayaka, O. Ito, S. Iijima and M. Yudasaka, Proc. Natl. Acad. Sci. U. S. A., 2008, 105, 14773.

8 J. W. Snyder, W. R. Greco, D. A. Bellnier, L. Vaughan and B. W. Henderson, Cancer Res., 2003, 63, 8126.

9 A. Khdair, C. Di, Y. Patil, L. Ma, Q. P. Dou, M. P. V. Shekhar and J. Panyam, J. Controlled Release, 2010, 141, 137.

10 Q. Yan, Y. Xin, R. Zhou, Y. Yin and J. Yuan, Chem. Commun., 2011, 47, 9594.

11 (a) L. Sun, X. Ma, C.-M. Dong, B. Zhu and X. Zhu, Biomacromolecules, 2012, 13, 3581; (b) G.-Y. Liu, C.-J. Chen, D.-D. Li, S.-S. Wang and J. Ji, J. Mater. Chem., 2012, 22, 16865.

12 G. Liu and C.-M. Dong, Biomacromolecules, 2012, 13, 1573.

13 J. Babin, M. Pelletier, M. Lepage, J.-F. Allard, D. Morris and Y. Zhao, Angew. Chem., Int. Ed., 2009, 48, 3329.

14 S. D. P. Baugh, Z. Yang, D. K. Leung, D. M. Wilson and R. Breslow, J. Am. Chem. Soc., 2001, 123, 12488.

15 I. Kraljić and S. E. Mohsni, Photochem. Photobiol., 1978, 28, 577.

16 J. Lee, C. Jeong and W. J. Kim, J. Mater. Chem. B, 2014, 2, 8338. 